\title{
Simulation of Velocity and Temperature fields
}

\author{
Mahmood Khalid Mawlood \\ Dep. of Mechanical Engineering, \\ College of Engineering, \\ University of Kirkuk
}

\author{
Ihsan Fadhil Abbas \\ Dep. of Refrigeration and \\ Air Conditioning, \\ College of Technology - Kirkuk
}

\begin{abstract}
In this work, a numerical method is developed for solving the unsteady NavierStokes and energy equations in a two-dimensional passive heating Trombe wall system. A parallel computer code that can be run efficiently on multicore PCs or networked workstations is developed for calculating the velocity and temperature fields in the whole domain of the system. The developed code is validated using data from the literature. Results are presented for the time response of a typical classical Trombe wall system to transient heating.

$$
\text { نمذجة السرع ودرجات الحرارة في جدار نزومب ذو بعدين }
$$

\section{الخلاصة}

لقد تم في هذه الدراسة تطوير طريقة عددية لحل معادلات نافير-ستوكس ومعادلة الطاقة لنظام التدفئة باستخدام جدار التبار

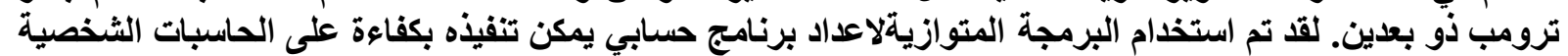

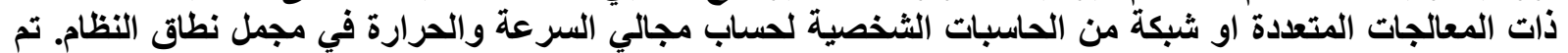

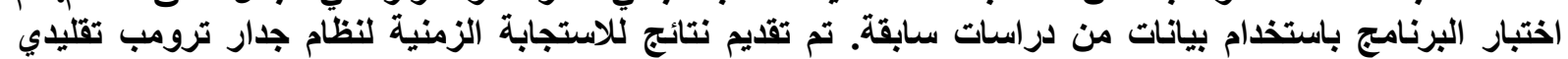
معرض لتسخين عابر.
\end{abstract}




\section{Introduction}

Passive heating Trombe wall systems have been the subject of a large number of experimental and numerical investigations during the past three decades. In the present review attention is paid to the numerical studies which are categorized here as truncated and full Trombe wall systems. By a truncated system it is meant that the study focuses on the motion and thermal behavior of the flow in the channel formed by the massive wall and theglazing without any interaction with the heated space. Full Trombe wall systems are those connected to a room through two air vents and the study takes into account the interaction between the room and the channel by the action of natural circulation. In addition, a full system may include an insulating partition between the massive walland glazing to form the so called 'composite' Trombe wall system.

Akbari and Borgers [1] and Borgers and Akbari [2] present two numerical studies for steady state laminar and turbulent flows respectively, between two vertical parallel plates to simulate the flow in a truncated Trombe wall system. The studies were based on the solution of the governing natural convection equations with the channel walls being at different constant temperatures and the temperature of the inlet air being known. A number of correlations were presented for calculating the performance of a Trombe wall.

Li et al. [3] performed steady state computations solving the governing equations by a finite volume method in a truncated Trombe channel. The massive wall was assumed to be heated by a constant heat flux on the side facing the glazing while the other side was assumed to be adiabatic. The glazing was assumed to be isothermal. The rate of heat transferred to the air that flows into the channel from the lower side vent was calculated for given wall heat fluxes and inlet air temperatures.

In an attempt to find correlations for the optimum wall spacing and the induced mass flow rate in truncated Trombe wall systems, Zamora and Kaiser [4] solved the natural convection equations and obtained simulations of steady two-dimensional laminar, transitional and turbulent flows with the channel walls being isothermal at different temperatures. The system configuration was slightly different than the above cited ones by that the side horizontal channels connected to the upper and lower vents were included in the computational domain.

The first prediction method of a full Trombe wall system seems to be that of Ormitson et al. [5] in which the channel, the room and inlet and outlet sections were all included. The channel walls and the room wall were assumed to be isothermal at different temperatures. Results for velocity and temperature distributions were reported for steady two-dimensional laminar flow. It was shown that the natural convection in the whole system was governed by an internal and external Rayleigh numbers.

Ben Yedder et al. [6] and Ben Yedder and Bilgen [7] developed a numerical method and performed computations for steady state laminar flow in full Trombe wall systems with isothermal walls [6] and with a constant heat flux [7]. Results for velocity and temperature fields were reported as well as Nusselt number for various Rayleigh numbers.

Mezrhab and Rabhi [8] performed computations for a full Trombe wall system. The mass flow rate and heat transfer properties due to natural convection in a two-dimensional cavity containing a Trombe wall type partition was investigated numerically. The effects of radiation and vent size on heat transfer were studied and steady state results for laminar flow at various Rayleigh numbers were reported.

In all the above cited papers the governing equations of natural convection, i.e. the continuity, momentum and energy equations, were solved numerically and detailed velocity and temperature fields were predicted for steady state cases only.

Unsteady or transient full Trombe wall systems have also gained considerable interest by researchers as the real Trombe walls are naturally unsteady systems. To study the performance of a novel Trombe wall with photovoltaic cells Jie et al. [9]developed an 
unsteady numerical model, performed computations and reported results for the variation of temperature of the system over a week of time using real meteorological data. Jie et al. [10] used a similar model to study the effect of selective thermo-insulation facades on the performance of a Trombe wall system and reported results of calculations for 24 hours. A similar model was developed by Shen et al. [11] to compare the thermal performances of the classical Trombe wall and a composite Trombe wall in cloudy and cold climates. Results were reported for 67 days with real meteorological input data.

All the unsteady numerical methods cited above and reviewed in this work are based on the solution of condensed parameter simplified equations and empirical correlations. The simplifying assumptions used in unsteady models render the computational methods less reliable, inaccurate and inappropriate for designing purposes.

The present work is motivated by the fact that all real Trombe wall systems are naturally unsteady and that the prediction of time evolution of the velocity and temperature fields of the entire system domain provides better insight in their design and application. Therefore, the present work aims at developing a computational method for the solution of the unsteady Navier-Stokes and energy equations throughout the whole system. To reduce computational time, parallel computing will be used to develop a code that can be run on multicore PCs or networked workstations.

The remainder of the paper is organized as follows: Section 2 describes the geometry of the physical problem and presents the mathematical model as well as the method of solution. Results and discussions are presented in Section 3 and conclusions in Section 4.

\section{System Description and Mathematical Model}

A schematic of a two-dimensional conventional Trombe wall system is shown in Figure (1). It is composed of a massive wall and glazing forming a solar collector connected to the heated room through two air vents. The incident solar radiation through the glazing is absorbed by the massive wall part of which is transmitted to the room by natural convection by the air circulating through the air vents. The other part of the absorbed energy is transmitted to the room by conduction.

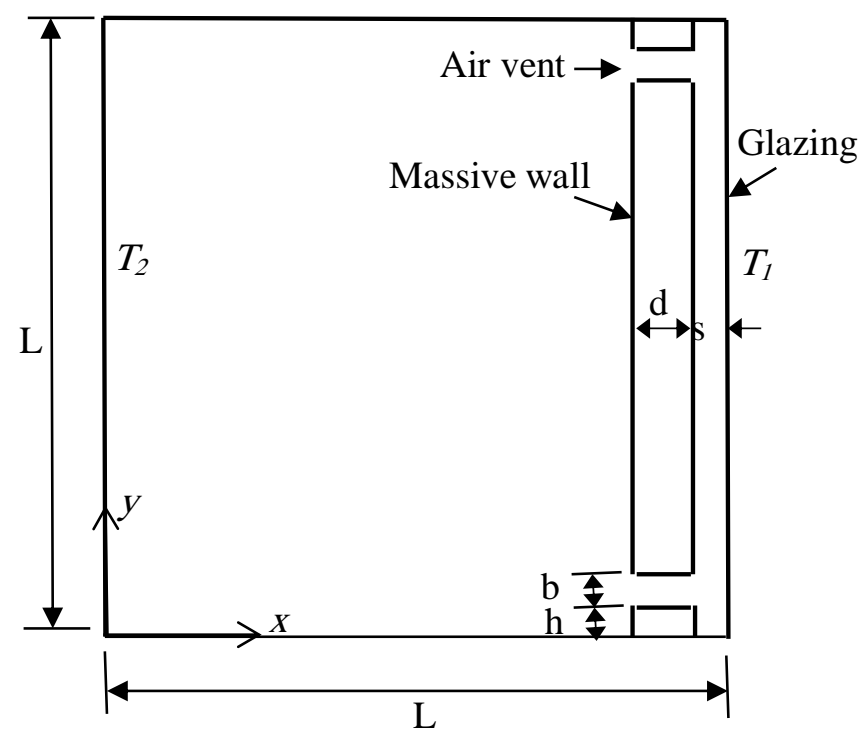

Figure 1. A Shematic of Trombe Wall System

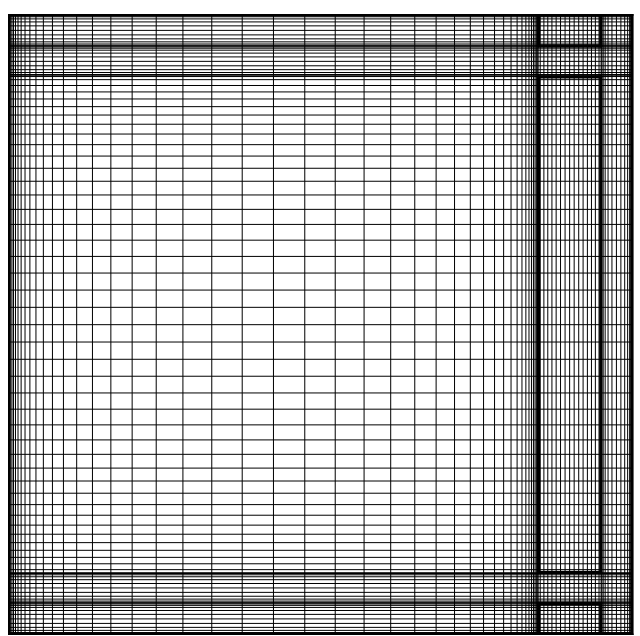

Figure 2. A Typical Computational Grid

The governing equations of the unsteady Trombe wall system with incompressible fluid flow can be written as 
Continuity equation:

$\nabla \cdot \mathbf{V}=0$

Momentum equations:

$\rho \frac{\partial \mathbf{V}}{\partial t}+\rho \mathbf{V} \cdot \nabla \mathbf{V}=\mathbf{f}-\nabla p+\nabla \cdot\left(\mu_{e f f} \nabla \mathbf{V}\right)$

Energy equation:

$\rho c \frac{\partial T}{\partial t}+\rho c \mathbf{V} \cdot \nabla T=\nabla \cdot\left(k_{e f f} \nabla T\right)+S$

In the above equations $c$ is the specific heat, $\mathbf{f}$ is the buoyant force, $k_{\text {eff }}$ is the effective thermal conductivity, $p$ is the pressure, $S$ is the volumetric rate of heat generation, $t$ is time, $T$ is the temperature, $\mathbf{V}$ is the velocity vector, $\mu_{\text {eff }}$ is the effective viscosity and $\rho$ is the density. The Boussinesq approximation is used to account for the source term $\mathbf{f}$ in the momentum equations.

The effective thermal conductivity for the fluid part $k_{\text {eff }}$ is calculated from

$k_{e f f}=k_{f}+\frac{v_{t}}{\sigma}$

and for the solid part from

$k_{\text {eff }}=k_{s}$

where $k_{f}$ and $k_{s}$ are the thermal conductivities of the fluid and the solid parts respectively, and $\sigma$ is the turbulent Prandtl number. The effective viscosity of the fluid is given by

$\mu_{e f f}=\mu+\rho v_{t}$

A simple zero-equation turbulence model [12] is used in this study to determine the turbulent eddy viscosity:

$v_{t}=C V l$

where $C$ is an empirical constant, $V$ is the local mean velocity and $l$ is the distance to the nearest solid wall.

The above governing equations (1) to (3) are solved for the whole system domain including both the fluid and the solid regions. Boundary conditions are imposed at the exterior solid surfaces. In the internal solid regions the temperature field is obtained from the solution of the energy equation while the velocity field is set to zero. The thermal conductivity at the solid-fluid interfaces is determined from local energy balance among the adjacent cells.

The numerical method used for solving the system of equations (1) to (3) is based on an explicit finite-volume method on a non-uniform structured grid. A typical stretched grid is shown in Figure 2 with higher node densities near the solid walls. To avoid unrealistic pressure oscillations the velocity components are calculated on a staggered grid [13]. 
The projection (or fractional step method) [14] in which the unsteady momentum equations are first advanced in time for computing an intermediate velocity field $\mathbf{V}^{+}$may be summarized as follows.

Equation (2) is written in a semi-discretized form as:

$$
\frac{\partial \mathbf{V}}{\partial t}=L\left(\mathbf{V}^{n}\right)
$$

where $L\left(\mathbf{V}^{n}\right)$ is a shorthand notation for the discretized convection, diffusion, pressure gradient and body force terms using the old values of all the dependent variable at the previous time step $n$.

In this study the standard four-step fourth-order Runge-Kutta method is used for time advancement of (8) to obtain the intermediate velocity $\mathbf{V}^{+}$

$$
\begin{gathered}
\mathbf{V}^{*}=\mathbf{V}^{n}+\frac{\Delta t}{2} L\left(\mathbf{V}^{n}\right) \\
\mathbf{V}^{* *}=\mathbf{V}^{n}+\frac{\Delta t}{2} L\left(\mathbf{V}^{*}\right) \\
\mathbf{V}^{* * *}=\mathbf{V}^{n}+\Delta t L\left(\mathbf{V}^{* *}\right) \\
\mathbf{V}^{+}=\mathbf{V}^{n}+\frac{\Delta t}{6}\left(L\left(\mathbf{V}^{n}\right)+2 L\left(\mathbf{V}^{*}\right)+2 L\left(\mathbf{V}^{* * *}\right)+L\left(\mathbf{V}^{* * * *}\right)\right)
\end{gathered}
$$

The intermediate velocity $\mathbf{V}^{+}$does not satisfy continuity equation (1). Therefore, the next step is to correct this velocity by the incompressibility constraint and solving a Poisson pressure correction equation of the form [15]

$$
\nabla^{2} p^{\prime}=\frac{\rho}{\Delta t} \nabla \cdot \mathbf{V}^{+}
$$

After having computed $p^{\prime}$ the pressure and velocity can be updated from

$$
\begin{aligned}
p^{n+1} & =p^{n}+p^{\prime} \\
\mathbf{V}^{n+1} & =\mathbf{V}^{+}-\frac{\Delta t}{\rho} \nabla p
\end{aligned}
$$

After the advancement of the momentum equation, the temperature field is computed by the same $4^{\text {th }}$-order RK method in time and a second-order finite-volume method in space. The time step $\Delta t$ is controlled by the stability requirement of the scheme. It is worth mentioning that the $4^{\text {th }}$-order RK method exhibits better stability properties over the simple forward scheme.

\subsection{Parallel Programming}

A parallel FORTRAN 90 code is developed for solving the governing equations in twospace dimensions using the above-described method of solution. The code can be run on a single multicore PC or a number of PCs networked together. The present code divides the 
computational domain, in the vertical y-direction only, into a number of sub-domains that equals the number of cores or compute nodes to be used in the calculation course. In order to balance the load among the CPU cores each sub-domain should have approximately the same number of computational mesh cells. The second-order spatial discretization scheme utilized in the code requires that any two adjacent sub-domains should overlap with each other at their interface boundaries by one layer of mesh cells. Message passing by the MPI [16] library routines are used for the exchange of data among the processes of the adjacent sub-domains. All the required initial data are input to the master node or master processor which are broadcast later to the other processors. Results obtained by the various processors at the end of the computation are sent back to the master node for output.

\section{Results and Discussions}

The developed code will first be validated by comparisons with data chosen from the literature pertaining to natural convection in a square cavity and a full Trombe wall systemand then the temporal behavior of a typical full Trombe wall system and the flywheel effect of the massive wall will be presented.

\subsection{Code Validation}

The buoyancy driven square cavity problem has been extensively used by researchers as a test case for validating computational methods $[17,18]$. This same problem is used here for testing the present method. Results of the present method for the average Nusselt number $\mathrm{Nu}$ as a function of Rayleigh number $R a$ in comparison with Refs. [17] and [18] are shown in Figure (3). The $N u$ and $R a$ numbers are defined by the following relations:

$$
\begin{gathered}
N u=-\left.\frac{L}{\left(T_{1}-T_{2}\right)} \int_{0}^{L} \frac{\partial T}{\partial x}\right|_{x=0} d y \\
R a=\frac{g \beta\left(T_{1}-T_{2}\right) L^{3} P r}{v^{2}}
\end{gathered}
$$

where $L$ is the side length of the square cavity, $T_{1}$ and $T_{2}$ are the temperatures of the side walls, $\beta$ is the volumetric expansion coefficient and $P r$ is the Prandtl number.

The range of $R a$ considered $\left(10^{3}-10^{10}\right)$ includes laminar and turbulent flow regimes. As in Ref. [18] it has been assumed that the flow becomes turbulent at $R a \geq 10^{8}$. The value of the empirical constant $C$ in Equation (7) is taken as 0.021. All the calculations were performed using $81 \times 81$ grid points. The comparison shows good agreement with the cited references.

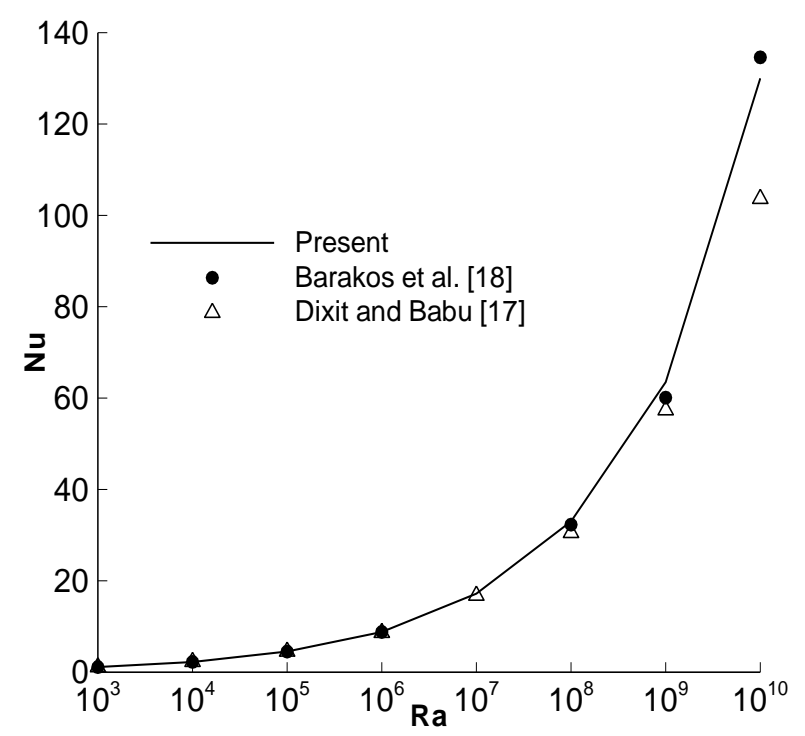

Figure 3. Comparison of results with Refs. [17] and [18] 
The second test problem is the steady state full Trombe wall system of Mezrhab and Rabhi [8]. The geometry of the problem considered is similar to that shown in Figure (1) with the following geometrical proportions:

$s / L=0.05, d / L=0.15, h / L=0.05$ and $b / L=0.15$.

The thermal properties of the system are as follows:

$\operatorname{Pr}=0.71, T_{1}-T_{2}=15 \mathrm{~K}, k_{s} / k_{f}=50$.

Mezrhab and Rabhi [8] performed computations for laminar flow and presented results for $\mathrm{Nu}$ with $\mathrm{Ra}$ varying from $10^{3}$ to $10^{7}$ and contour plots of streamlines and isotherms for $R a=$ $10^{6}$. In the present test, calculations are performed for $R a=10^{6}$ and $10^{7}$ for comparison purposes.

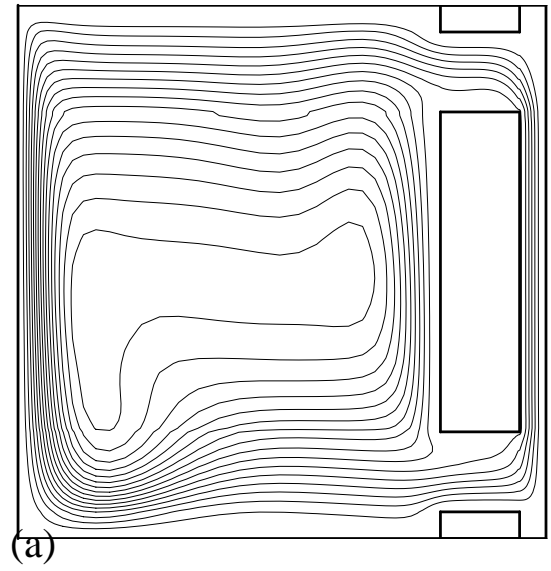

Figure 4.Plots of streamlines (a) and isotherms (b) for $\mathrm{Ra}=10^{6}$

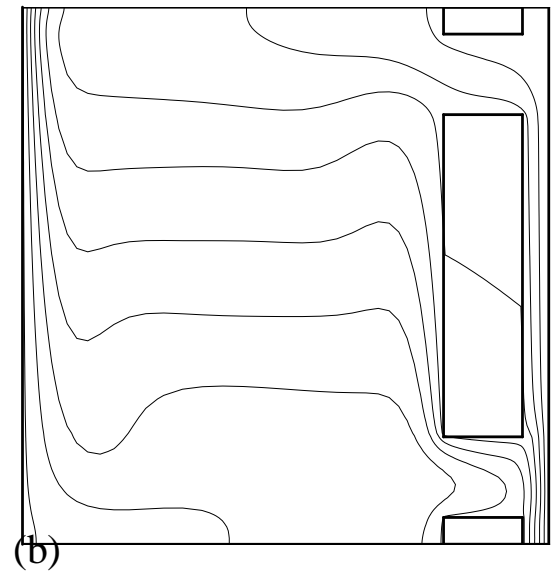

Figures $4 \mathrm{a}$ and $4 \mathrm{~b}$ show the contour plots of the streamlines and isotherms respectively for $R a=10^{6}$. Qualitatively the present calculations reproduce the plots presented in Ref. [8].

Quantitative comparison of results for Nusselt number $N u$ and the normalized flow rate $Q$ of air circulating through the vents is shown in Table 1. The normalized flow rate is calculated from

$Q=\frac{1}{\alpha} \int_{h}^{(h+b)} u d y$

where $\alpha$ is the thermal diffusivity defined as $\alpha=k / \rho c$.It can be seen that the results are in good agreement.

Table 1. Comparison of results with Mezrhab and Rabhi [8]

\begin{tabular}{|l|c|c|c||}
\hline \multicolumn{2}{|c|}{$R a$} & $10^{6}$ & $10^{7}$ \\
\hline \hline Present results & $N u$ & 7.28 & 15.26 \\
& $Q$ & 5.01 & 21.47 \\
\hline Mezrhab and Rabhi [8] & $N u^{+}$ & 7.2 & 14.5 \\
& $Q^{+}$ & 4.25 & 19.5 \\
\hline
\end{tabular}

${ }^{+}$These values are extracted from small plots of Ref. [8] 


\subsection{A Typical Transient System}

The transient response of a typical Trombe wall system with the following geometric proportions will be considered here.

$\frac{s}{L}=\frac{h}{L}=\frac{b}{L}=0.1 ; \frac{d}{L}=0.2$

An attempt will be made to show the flywheel effect of the massive wall on the transient behavior of the system. Therefore, two massive walls will be considered, a heavy one made of concrete and a light one having similar thermal properties as the heavy one except its density. Thermo-physical properties of the heavy massive wall (concrete) relative to that of air are:

$\frac{k_{s}}{k_{f}}=60 ; \frac{\rho_{s}}{\rho_{f}}=1650 ; \frac{c_{s}}{c_{f}}=1$

and for the light massive wall

$\frac{\rho_{s}}{\rho_{f}}=100$

For the initial conditions at time $t=0$, the whole domain is assumed be at a given initial temperature $T_{i}$ and the air to be stagnant. For time $t>0$, the boundary conditions and the heat received by the massive wall are prescribed as follows:

Left wall and the glazing:

$u=v=0 ; T_{1}=T_{2}=T_{i}$

where $u$ and $v$ are the horizontal and vertical velocity components.

Top and bottom walls:

$u=v=0 ; \frac{\partial T}{\partial y}=0$

The massive wall receives heat according to the following relations:

$q=q_{\max } \sin \left(\frac{2 \pi t}{3600}\right) ; 0<t \leq 3600 s$

$q=0 ; \quad t>3600$ s.

which is a half sine wave applied over a period of one hour. The average heat flux over the heating period received by the massive wall becomes

$\mathrm{q}_{\mathrm{av}}=2 \mathrm{q}_{\max } / \pi$

The Rayleigh number $R a_{q}$ based on the maximum heat flux $q_{\max }$ of this problem is $3.5 \times 10^{13}$ where $R a_{q}$ is defined as

$R a_{q}=\frac{g \beta L^{4} q_{\max }}{v \alpha k}$

Computations are performed over a period of three hours. Figure 5 shows the variation of the temperature measured at the level $y=L / 2$ on the left and right sides of the massive wall and at the center of the heated space. For this same wall the heat loss from the system calculated as heat flux normalized with $q_{a v}$ is shown in Figure 6. The computations are repeated for the light massive wall and the results are shown in Figures 7 and 8. Comparison of the two sets of results shows that the temperature of the system with light massive wall increases at faster rates than that with heavy massive wall. The heat transfer through the light wall due to conduction is much faster than that of the heavy wall which causes the temperature on both sides of the lighter wall approach the same value a short period after the end of heating process. Due to this fast increase in temperature the heat loss from the lighter system is also 
higher than the heavy system as seen from Figure 8 . Thus a system with lighter massive wall will be driven faster to reach steady state than a system with heavy wall.

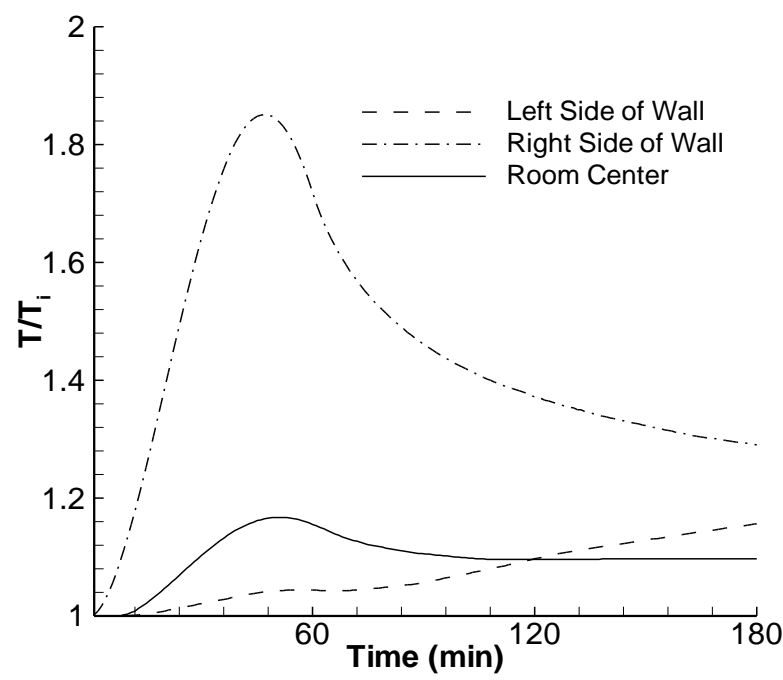

Figure 5. Variation of heavy-wall system temperature with time.

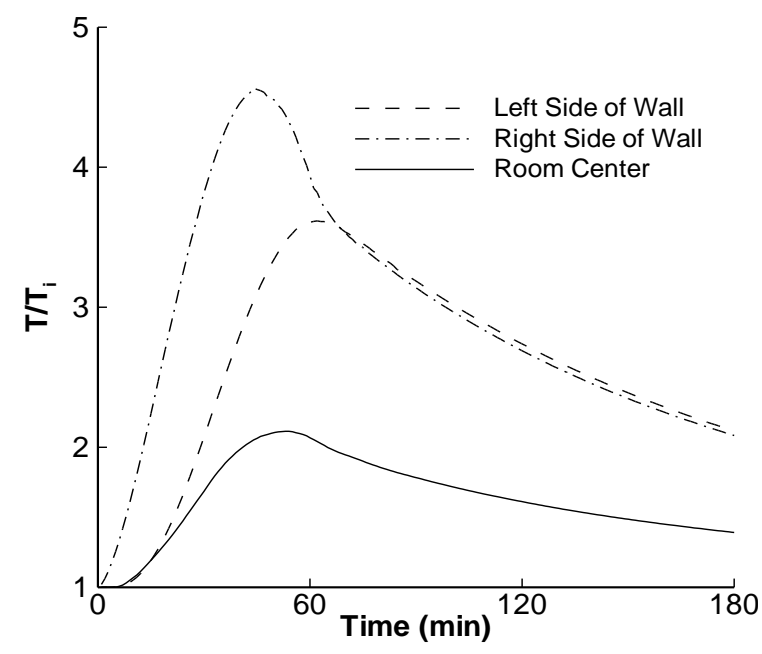

Figure 7. Variation of temperature of the light-wall system with time.

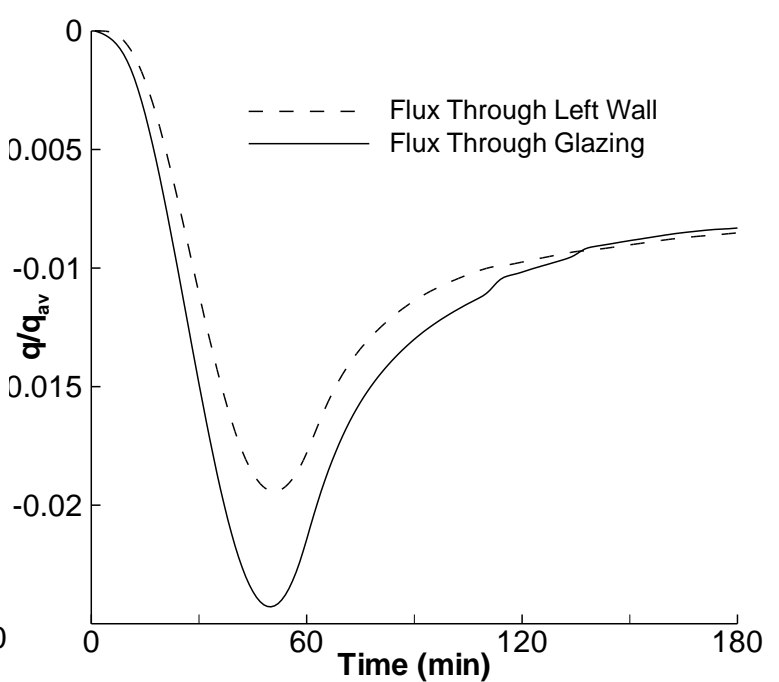

Figure 6. Variation of heat loss from the heavy-wall system with time.

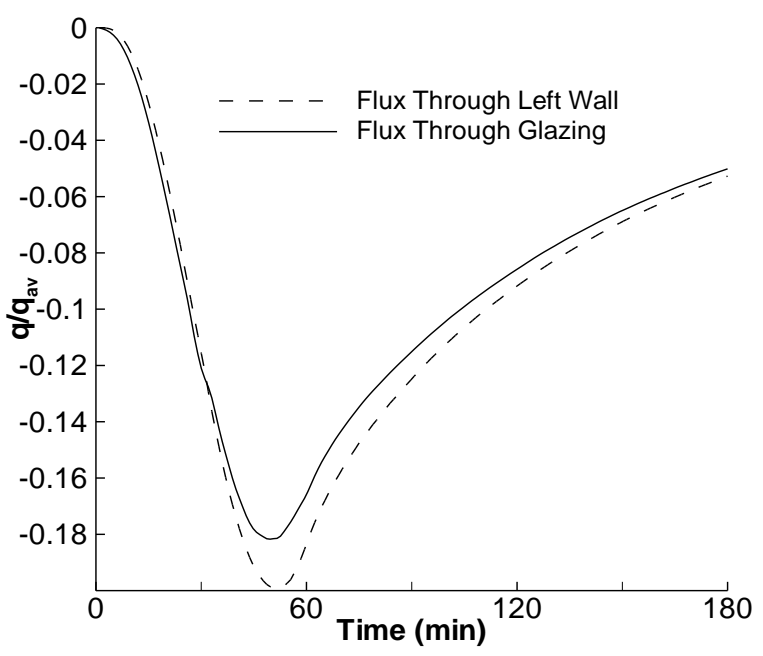

Figure 8. Variation of heat loss from the light-wall system with time.

Another important variable that attention must be paid to is the induced flow rate of air that circulates in the system as a result of buoyancy. Figure 9 shows the variation of the normalized flow rate $Q$ through the system with time for both the heavy and light wall systems. It can be seen that due higher temperature gradients in the light wall system the induced flow rate steeply rises to higher values at the beginning of the heating process, steeply drops as the heating rate starts to decrease and almost stagnant after a short period after ending the heating process. While in the heavy wall system the initial rise of the flow rate is less steep and flat over some time during the heating period and then gradually drops after the end of heating period. 
During the computation process values of detailed field variables are stored at prescribed intervals of time to enable tracking the behavior at every individual location of the system. As an example, time station $t=30$ minutes, is chosen to view the flow pattern and temperature distribution throughout the system as shown in Figures 10 and 11.

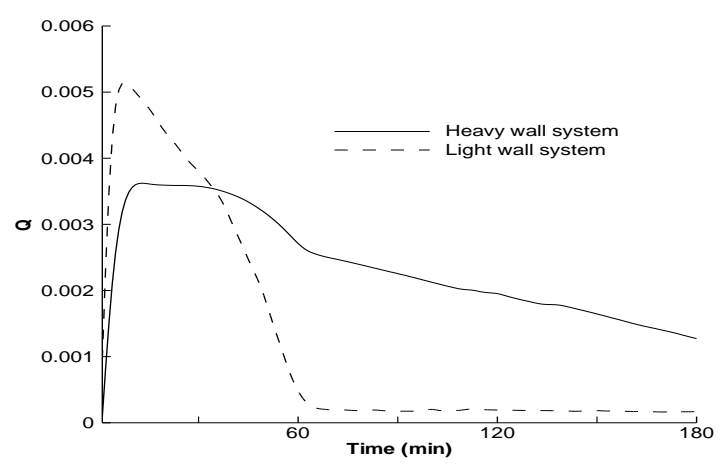

Figure 9. Normalized flowrate through the system.

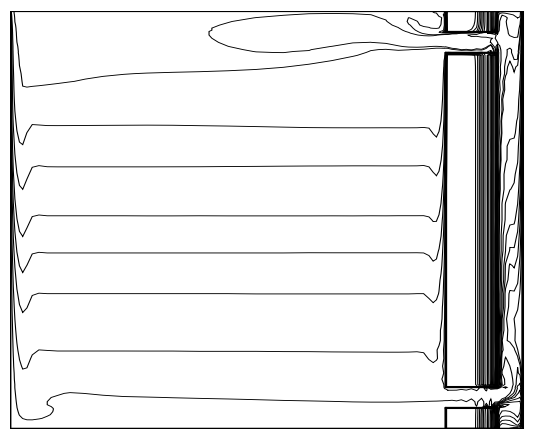

Figure 11. Isotherms of the heavy wall system at time $t=30 \mathrm{~min}$.

The above discussed behavior of the two systems may bring up a number of questions to be asked about the optimum design of a Trombe wall system, the material to be selected, the optimum geometrical proportions, vent sizes and their location, etc. Such design requirements can only be fulfilled by extensive parametric studies of the system, and such studies can only be performed by efficient computational tools like the code developed in this work.

\subsection{Code Efficiency}

The efficiency of the developed parallel code is evaluated by calculating the speedup of the code which is defined as

$S t=t_{s} / t_{p}$

where $t_{s}$ and $t_{p}$ are the CPU times of a serial and a parallel code respectively.

The code is run on a PC having 8-cores(Intel® Corei7 - 2.2 GHz). Figure 12 shows results of the speedup of the computational system for a grid size of $91 \times 91$ points. 


\section{Conclusions}

A numerical method and an efficient parallel code are developed for solving the unsteady Navier-Stokes and energy equations in two-dimensional Trombe wall system. The code is validated using data from the literature. The temporal behavior of a typical transient Trombe wall system is studied. The flywheel effect of the massive wall on the time response is demonstrated by considering a heavy and a light wall system. The study shows that an optimum design of a passive heating Trombe wall system requires extensive parametric investigation by efficient computational tools such as the one developed in this work.

\section{References}

[1]Akbari, H. and Borgers, T. R., "Free Convective Laminar Flow within the Trombe Wall Channel", Solar Energy, Vol. 22, 1979, pp. 165-174.

[2]Borgers, T. R. and Akbari, H., "Free Convective Turbulent Flow within the Trombe Wall Channel", Solar Energy, Vol. 28, No. 3/4, 1982, pp. 253-264.

[3] Li, Y., Duanmu, X., Sun, Y., Li, J., and Jia, H., " Study on the Air Movement Character in Solar Wall System", Proceedings: Building Simulation 2007, pp. 927-931.

[4] Zamora, B., and Kaiser, A. S., "Thermal and Dynamic Optimization of the Convective Flow in Trombe Wall Shaped Channels by Numerical Investigation", Heat Mass Transfer, Vol. 45, 2009, pp. 1393-1407.

[5] Ormitson, S. J., Raithby, G. D., and Hollands, K. G. T., "Numerical Predictions of Natural Convection in a Trombe Wall System", Int. J. Heat Mass Transfer, Vol. 29, No. 6, 1986, pp. 869-877.

[6] Ben Yedder, R., Du, Z. G., and Bilgen, E., "Numerical Study of Laminar Natural Convection in Composite Trombe Wall Systems", Solar and Wind Technology, Vol. 7, No. 6, 1990, pp. 675-683.

[7] Ben Yedder, R., and Bilgen, E., "Natural Convection and Conduction in Trombe Wall Systems", Int. J. Heat Mass Transfer, Vol. 34, No. 4/5, 1991, pp. 1237-1248.

[8] Mezrhab, A., and Rabhi, M., "Modeling of the Thermal Transfers in an Enclosure of the Trombe Wall Type", Int. Sci. J. Alternative Energy and Ecology, Vol. 62, No. 6, 2008, pp. 9-14.

[9] Jie, J., Hua, Y., Wei, H., Gang, P., Jianping, L., and Bin, J., "Modeling of a Novel Trombe Wall with PVC Cells", Building and Environment, Vol. 42, 2007, pp. 1544-1552. [10] Jie, J., ChengLong, L., Wei, S., HanCheng, Y., Wei, H. and Gang, P., "An Improved Approach for the Application of Trombe Wall System to Building Construction with Selective Thermo-Insulation Facades", Chinese Science Bulletin, 2009, pp.1949-1956.

[11] Shen, J., Lassue, S., Zalewski, L., and Huang, D., "Numerical Study on Thermal Behavior of Classical or Composite Trombe Solar Walls", Energy and Buildings, Vol. 39, 2007, pp. 962-974.

[12] Chen, Q., and Xu, W., "A Zero-Equation Turbulence Model for Indoor Air Flow Simulation", Energy and Buildings, Vol. 28, No. 2, 1998, pp. 137-144.

[13] Patankar, S. V.,"Numerical Heat Transfer and Fluid Flow", Hemisphere, Washington, DC, 1980 .

[14] Kim, J., and Moin, P., "Application of a Fractional-StepScheme for the Incompressible Navier-Stokes Equations,"J. Comput. Phys., Vol. 56, 308-323, (1985).

[15] Tannehill, J. C., Anderson, D. A. and Pletcher, R. H., "Computational Fluid Mechanics and Heat Transfer", Taylor\& Francis, (1997).

[16] Gropp, W,. Lusk, E., and Skjellum, A, "Using MPI:Portable Parallel Programming with 
the Message PassingInterface", Cambridge: MIT Press, 1994.

[17] Dixit, H. N., and Babu, V., "Simulation of High Rayleigh Number Natural Convection in Square Cavity Using the Lattice Boltzmann Method", Int. J. Heat Mass Transfer, Vol. 49, 2006, pp. 727-739.

[18] Barakos, G., Mitsoulis, E., and Assimacoulos, D., "Natural Convection Flow in a Square Cavity Revised: Laminar and Turbulent Models with Wall Functions", Int. J. Num. Meth.

Fluids, Vol. 18, 1994, pp. 695-719. 